\title{
Partial penetrance keeps the options open
}

Evolution often occurs in discrete steps, but the genetic pathways to different phenotypes and the intermediate steps that allow evolutionary changes to occur are often unknown. Writing in Nature, Eldar, Chary and colleagues show that mutations with partial penetrance can enable a gradual transition between two phenotypes.

Bacillus subtilis cells form spores under unfavourable conditions. Sporulation starts with the formation of a septum that asymmetrically separates the cell - one chromosome is segregated into the smaller compartment, the forespore, while the other chromosome remains in the mother cell. If two asymmetric septa are formed, the chromosomes are distributed to the two forespores, leaving the mother cell without a chromosome. These 'abortively disporic' cells do not form viable spores. Normally, the signalling protein SpoIIR signals

Partial penetrance in Bacillus subtilis sporulation. All cells contain a mutation in the spollR gene but are otherwise genetically identical. Some of the cells develop normally, producing one spore per cell (red brackets), whereas others undergo twin sporulation, producing two spores per cell (blue bracket). Spores can be seen as white objects in the darker body of the cell. Also shown is a membrane dye (green), which strongly marks spores and septa. Note the absence of the septum in the twin-sporulating cell. Image courtesy of A. Eldar, California Institute of Technology, USA. from the forespore to the mother cell to prevent the formation of these abortively disporic cells.

Eldar and colleagues noticed that mutations that alter the timing or levels of expression of spoIIR result in partial penetrance, and B. subtilis cells harbouring these mutations display a range of phenotypes, including normal sporulation, the abortive disporic phenotype and an 'escape' phenotype that leads to twin sporulation (the formation of two viable spores inside a single mother cell) (see the figure). Further investigations revealed that the fate of individual B. subtilis cells is only partially regulated by SpoIIR.

To make two viable spores, the bacterium must make additional septa and over-replicate its chromosome compared with wild-type cells. The authors tested whether the predisposition of cells to form twin spores could be increased by genetically altering these properties. Increasing levels of SpoIIE, a protein that controls septation during sporulation, caused a decrease in the escape phenotype compared with the abortive disporic phenotype. The number of chromosomes present during sporulation could be increased by mutations that affect chromosome replication (mutations in $y a b A$ and a hypomorphic allele of spo0J). The fitness cost of the increased chromosome number was slight - these mutants sporulated at a frequency of $75 \%$ of that of wild-type cells - and the increased chromosome number induced only a low level of twin sporulation. However, when these mutations were combined with the spoIIR mutations, the number of twin-sporulating cells increased significantly, reaching levels seen in species that undergo twin sporulation naturally. The authors went on to analyse natural twin sporulation in Clostridium oceanicum and showed that the order of replication and septations as well as the duration between septations are the same as in the B. subtilis twin mutants, further strengthening the evolutionary relevance of their analysis.

This study shows that partial penetrance can prime a cell for the acquisition of new phenotypes. A single mutation with partial penetrance, in this case in spoIIR, allows normal sporulation to occur while producing an alternative fate in a small subset of cells. When this is combined with a second mutation, in this case a yabA mutation, the penetrance of the first mutation is greatly increased. With each mutation, the cell does not commit to a unique fate but opens the door to new outcomes, which can be enhanced by additional mutations.

Christiaan van Ooij, Chief Editor, Nature Reviews Microbiology

ORIGINAL RESEARCH PAPER Eldar, A. et al.

Partial penetrance facilitates developmental evolution in bacteria. Nature 460, 510-514 (2009) 